registry catchment areas was determined. Standardised incidence ratios (SIR) and standardised rate ratios for bladder cancer were calculated by exposure category and cumulative rank quartiles for different lag periods. Cox regression was used to model bladder cancer incidence with estimated cumulative rank, adjusting for confounders. Indirect methods were used to control for smoking. Results Excess bladder cancer was observed compared to the New York State population (SIR $=2.87,95 \%$ confidence interval $[\mathrm{CI}]$ 2.02-3.96), with higher elevations among workers definitely exposed (moderate/high) (SIR $=3.90,95 \%$ CI 2.57-5.68) and in the highest cumulative rank quartile $(\mathrm{SIR}=6.13,95 \%$ CI 2.80 11.6, 10-year lag). Bladder cancer rates increased significantly with estimated cumulative rank (10-year lag). Smoking only accounted for an estimated $8 \%$ elevation in bladder cancer incidence.

Conclusions Bladder cancer incidence remains elevated in this cohort and significantly associated with estimated cumulative exposure. Results are consistent with earlier findings in this and other cohorts. Despite other concurrent chemical exposures, we consider o-toluidine most likely responsible for the bladder cancer incidence elevation and recommend a reexamination of occupational exposure limits.

\section{MULTIMORBIDITY AND PREVIOUS SICKNESS ABSENCE EPISODES ARE DETERMINANTS OF INCIDENCE AND DURATION OF FUTURE EPISODES}

\footnotetext{
1,2 Monica Ubalde-Lopez, ${ }^{1,3}$ David Gimeno, ${ }^{1,3}$ George L Delclos, ${ }^{4}$ Eva Calvo-Bonacho, 1,2 Fernando G Benavides. 'CISAL-Center for Research in Occupational Health, University Pompeu Fabra, Barcelona, Spain; ' $C I B E R E S P, C I B E R$ in Epidemiology and Public Health, Madrid, Spain; ${ }^{3}$ The University of Texas School of Public Health, Texas, USA; ${ }^{4}$ Ibermutuamur, Madrid, Spain
}

\subsection{6/oemed-2014-102362.30}

Objectives While sociodemographic and work-related factors are frequently studied as determinants of sickness absence (SA), health-related determinants have surprisingly received little attention. We examined the effect of multimorbidity and previous SA on the incidence and duration of future SA.

Method A retrospective (2004-2008) cohort of 373,905 workers who underwent a standardised medical evaluation in 2006 from which information on chronic conditions, health-related symptoms and behaviours was used to construct a sex-specific multimorbidity score (MMBS). Information on SA episodes occurring during the two years prior to the examination came from the employment history. We estimated the effect of the MMBS and prior SA on the 2-year incidence and duration of SA post-examination using a Cox model adjusted for age and occupational social class. Effects on SA duration were also adjusted for diagnosis.

Results Men, but not women, showed an effect with a trend of higher SA incidence risk from low $(\mathrm{HR}=1.06$; 95\% CI: $1.01-$ 1.11) to high MMBS (HR=1.22; 95\% CI: 1.18-1.28). Having five or more prior episodes was related to higher SA incidence risk, both in men ( $\mathrm{HR}=2.19$ 95\% CI: 2.11-2.28) and in women (HR $=2.47 ; 95 \%$ CI: 2.35-2.61). Women, but not men, had longer SA duration from low $(\mathrm{HR}=0.91 ; 95 \% \mathrm{CI}: 0.83-0.99)$ to high MMBS (HR $=0.88 ; 95 \%$ CI: $0.78-0.99)$. Having 5 or more prior $\mathrm{SA}$ episodes was related to shorter duration in men $(\mathrm{HR}=1.67$; 95\% CI: 1.30-2.16) and women ( $\mathrm{HR}=2.12$; 95\% CI: 1.56-2.89). Conclusions Multimorbidity increases the risk of higher SA incidence and duration while the effect of prior SA episodes is more complex.

\section{6}

MARGINAL STRUCTURAL MODELS FOR RISK OR PREVALENCE RATIOS AND DIFFERENCES

David Richardson. UNC, Chapel Hill, NC, USA

10.1136/oemed-2014-102362.31

Objectives Occupational epidemiologists often analyse binary outcomes in cohort and cross-sectional studies using multivariable logistic regression models, yielding estimates of adjusted odds ratios. When the outcome is common the adjusted odds ratio will not closely approximate the covariate-adjusted risk or prevalence ratio. Consequently, investigators may decide to directly estimate the risk or prevalence ratio using a log-binomial regression model; however, such models tend to be unstable and may not converge.

Method A marginal structural log-binomial model can be used to estimate risk and prevalence ratios and differences. The approach reduces problems with model convergence typical of log-binomial regression by shifting all explanatory variables except the exposures of primary interest from the linear predictor of the outcome regression model to a propensity score model for the exposure. The approach also facilitates evaluation of departures from additivity in the joint effects of two exposures.

Results We illustrate the proposed approach using data from several illustrative occupational studies of common outcomes. Conclusions The proposed approach facilitates analysis of risk or prevalence ratios and differences in cohort and cross-sectional studies with common outcomes.

\section{MATCHING AND COUNTER-MATCHING ON PROPENSITY SCORES IN NESTED CASE-CONTROL STUDIES}

David Richardson. UNC, Chapel Hill, NC, USA

10.1136/oemed-2014-102362.32

Objectives Occupational cohort studies often collect information on a primary exposure of interest and a small set of potential confounders. A nested case-control study may be undertaken to collect additional information on covariates that are potential confounders. Method We describe and illustrate an approach to matched nested case-control studies in such settings. The approach reduces problems of sparse data typical of matched nested casecontrol studies by reducing the set of matching on explanatory variables to a single dimension defined by a propensity score model for the exposure. We then consider additional uses of propensity score for matching. We first consider varying control: case ratio in matched designs to increase efficiency when exposure prevalence is rare. Next we consider a second propensity score that include additional covariates not in the set of strong confounders selected for matching. Counter matching on this score can further increase efficiency.

Results We illustrate the proposed approach using data from a cohort study of underground miners.

Conclusions The proposed approach can improve efficiency of nested case control designs.

\section{RACIAL AND GENDER DIFFERENCES IN THE RISK OF ISCHAEMIC HEART DISEASE AND THE HEALTHY WORKER SURVIVOR EFFECT AMONG AUTOWORKERS}

Sadie Costello, Sally Picciotto, Ellen Eisen. University of California, Berkeley, CA, USA

10.1136/oemed-2014-102362.33 
Objectives To examine gender and racial disparities in heart disease mortality related to metalworking fluid exposures and in the healthy worker survivor effect.

Method We examined ischaemic heart disease (IHD) mortality from 1941 to 1995 in a cohort of autoworkers with quantitative exposure to cumulative respirable particulate matter from waterbased metalworking fluids. Cox models were used to estimate the exposure-response to soluble and synthetic fluids separately in white men, black men, and white women. In separate analyses, we used g-estimation to adjust for the healthy worker survivor effect.

Results The risk of IHD was increased among black men (295 deaths) exposed to synthetic fluid with a hazard ratio (HR) of 3.47 (95\% CI: $1.52,7.92)$ in the highest cumulative exposure category. White women (119 deaths) had increased risk of IHD with increased soluble fluid (HR: $2.44(0.93,6.38)$ ) in the second to highest category. However, Cox models show no increased risk in white men (2246 deaths). In contrast, g-estimation results indicate that if white men had been always unexposed to soluble and synthetic fluid, then on average for each case, 2.99 and 2.77 years of life would have been saved, respectively.

Conclusions We found increased risk of IHD for black men and white women exposed to metalworking fluids using Cox regression. After adjusting for the healthy worker survivor effect, increased risk was observed for white men. The ability to leave work for health related reasons may be an option more available to white male workers.

\section{FARM EXPOSURES, ALLERGY SYMPTOMS AND RISK OF NON-HODGKIN LYMPHOMA IN THE AGRICULTURAL HEALTH STUDY}

${ }^{1} J$ onathan Hofmann, ${ }^{2}$ Jane Hoppin, ${ }^{1}$ Aaron Blair, ${ }^{1}$ Michael Alavanja, 'Laura Beane Freeman. ${ }^{1}$ National Cancer Institute, Bethesda, MD, USA; ${ }^{2}$ North Carolina State University, Raleigh, NC, USA

\subsection{6/oemed-2014-102362.34}

Objectives Exposure to allergens and microorganisms in the agricultural environment has been linked to altered immune response. Studies in the general population have reported reduced risks of non-Hodgkin lymphoma (NHL) among those with a history of atopic conditions, although results are inconsistent. To evaluate the allergy-NHL association in the context of farm exposures, we conducted an investigation in the Agricultural Health Study, a prospective cohort of farmers and spouses from North Carolina and Iowa.

Method Our study included 49656 farmers and spouses with crop and animal exposures and allergy symptoms reported at baseline (1993-1997). We identified 418 incident cases of NHL (including chronic lymphocytic leukaemia and multiple myeloma) during follow-up through 2010 in North Carolina and 2011 in Iowa. Hazard ratios (HR) and 95\% confidence intervals (CI) were calculated using multivariable-adjusted proportional hazards models.

Results At enrollment, over $80 \%$ of the study participants lived on farms growing grains or hay and $64 \%$ on farms raising livestock. Compared to individuals without allergy symptoms, those with symptoms had a reduced risk of NHL $(\mathrm{HR}=0.61,95 \%$ $\mathrm{CI}=0.50-0.74)$. We observed a slightly greater reduction in NHL risk among participants whose allergy symptoms worsened after working with grains and hay $(\mathrm{HR}=0.53,95 \% \mathrm{CI}=0.41-$ $0.69)$. The association between livestock and NHL was borderline significant overall $(\mathrm{HR}=0.82,95 \% \mathrm{CI}=0.66-1.01)$, and significant among those without allergy symptoms $(\mathrm{HR}=0.70$, $95 \% \mathrm{CI}=0.51-0.96)$.

Conclusions Our findings suggest that among individuals working and living on farms, allergy symptoms are associated with a reduced risk of developing NHL, and that risk may be influenced by particular farm characteristics.

\section{REDUCTION OF OCCUPATIONAL ACCIDENTS: EVIDENCE BASED PREVENTION AND THE PREVENTION INDEX (PI-TOP)}

${ }^{1}$ Frank Bochmann, ${ }^{2}$ Martin Arning, ${ }^{1} Y$ Y Sun, ${ }^{2}$ Jutta Boerger. ${ }^{1}$ IFA-Institute for Occupational Safety and Health of the German Social Accident Insurance, Sankt Augustin, Germany; ${ }^{2}$ German Social Accident Insurance Institution for the Woodworking and Metalworking Industries, Mainz, Germany

\subsection{6/oemed-2014-102362.35}

Objectives Prevention Index (PI-TOP) is a practical measurement tool which is currently used to monitor injury prevention activities at workplaces. This 12 -item scoring system has 3 subscales rating technical, organisational and personnel-related internal safety conditions of a company. The reliability and validity of this instrument were evaluated in a cross-sectional survey in the German metal industry during the time between December 2011 and May 2012.

Method The inter-rater-reliability of this instrument was examined by 2 trained supervisors of the German Social Accident Insurance Institution for the Woodworking and Metalworking Industries in 128 companies. The agreement of the double ratings was quantified by interclass correlation coefficient (ICC) and absolute agreement of the rating values. Construct validity of the score was examined by principle component factor analysis while content validity was evaluated by quantifying the association between PITOP-values and 5-years injury rates of 35000 companies. Poisson regression analysis was performed to assess the strength of the association adjusted for company size and related industrial sectors.

Results Our analysis indicate a moderate to good inter-rater-reliability (ICC $=0.46-0.75$ ) of PITOP-values with an absolute agreement between $72 \%$ and $81 \%$.

Factor analysis identified three component subscales which meet exactly the structural measure theory behind the score.

The Poisson regression analysis demonstrates that PITOP-values $\geq 7$ in the T-, $\mathrm{O}$ - and P-subscales are associated with a decrease of injury rates.

Conclusions Because this analysis indicates that PITOP is a valid and reliable instrument, it will be used to monitor safety conditions at workplaces in a longitudinal practical approach.

\section{PERSONAL ASSISTANCE - A PREREQUISITE TO WORK FOR PERSONS WITH SUBSTANTIAL AND PERMANENT DISABILITY?}

1,2leva Reine. 'Uppsala University, Uppsala, Sweden; '2Social Insurance Agency, Stockholm, Sweden

10.1136/oemed-2014-102362.36

Objectives In Sweden, people with a substantial and permanent disability have been entitled to personal assistance (PA) since the reform took effect in 1994. To qualify for government attendance allowance, a person must belong to one of the groups predefined in the Act Concerning Support and Service for Persons 